\title{
LCA in Strategic Decision Making for Long Term Urban Transportation System Transformation
}

\author{
Florian Ansgar Jaeger, Katrin Müller, Cornelia Petermann \\ and Eric Lesage
}

\begin{abstract}
The paper provides an overview of how Siemens uses LCA methodology and tools to support cities in the decision-making process to promote sustainable urban transportation systems. It focuses on GHGs and local air pollution. Determining the cause of GHG emissions and air pollution requires flexible scopes and a highly parameterized, hierarchical model, which can be adapted to any city's transportation system. Emission forecasting capabilities are very important since motorized transportation modes quickly change properties over time. The model screens a large set of infrastructure improvement measures by the click of a button and analyses their impact on KPIs for different years. Applicability, challenges and limits of LCA to the specific application of urban transportation system modelling are discussed.
\end{abstract}

\section{Introduction}

LCA methodology and tools have proven useful for assessing products or even companies in organizational LCA. Having used LCA-based methods and tools to model transport systems in more than 20 cities, we can say that this applies to mobility related infrastructure solutions in cities as well. This report shows how it was done and that the variety of indicators and flexibility LCA offers, not only matters to different products and product categories, but is also crucial for cities. The cities in the world have different focuses on environmental, economic or social

\footnotetext{
F. A. Jaeger $(\bowtie) \cdot$ K. Müller · C. Petermann

Research in Energy and Electronics, Energy Systems,

Sustainable Life Cycle Management and Environmental

Performance Management, Siemens Corporate Technology,

13629 Berlin, Germany

e-mail: florian_ansgar.jaeger@siemens.com

E. Lesage

Business Development and Strategy, Mobility Consulting,

Siemens Mobility, Berlin, Germany

(C) The Author(s) 2018

E. Benetto et al. (eds.), Designing Sustainable Technologies,

Products and Policies, https://doi.org/10.1007/978-3-319-66981-6_22
} 
aspects/KPIs. These differ by region, in scope (Carbon Accounting Scope 1-3) and in time (in terms of target setting, short term for air pollutants, long term for GHG-emissions). This diversity and the fact that there are vast differences in the level of maturity of cities with regard to data availability, setting targets and developing plans on how to achieve them, sets the requirements for any community scale model.

The aim of the transportation model is to support cities in creating climate action plans or clean air plans and to support cross-department activities in city administrations. The model is embedded in a process that supports city administrations in building alternative future infrastructure scenarios. It reflects all generally accepted/ assumed changes in technology, fleets and national policy over time by default and focus on the cities' core indicators.

\section{Method}

The methods and tools are a combination of conventional LCA, consequential LCA, greenhouse gas accounting for cities and futurology. Even though the software and modelling concept such as the functional units are common LCA-based, the model is aligned with the GPC-Protocol [1]. Forecasting elements are included from HBEFA [2] for market and technology development. Scenario workshops enable the inclusion of the political agenda during the projects process.

\subsection{Scope and Indicators}

The scope of the transport system model follows the basic rules of the GPC-Protocol [1], since greenhouse gas accounting is a quite common procedure for municipalities. It is a comparably well-defined process. The majority of cities with which we have worked have experts with a good understanding of the GPC-Protocol methodology and relevant stakeholders in cities are likely to know how to interpret its results.

With regard to the scope of activities, traffic in a territory is included, while emissions from airports and liquids or gases in pipelines are excluded. All processes are separated into the three scopes: direct, energy related indirect and other indirect emissions. By the click of a button, the model enables the inclusion and exclusion of different lifecycle phases or infrastructure categories and activities such as e.g. maintenance.

Despite these options being available, all cities we worked with decided to balance the direct operation of vehicles and direct emissions of infrastructure such as street lighting only. In addition to the standard activity data necessary to establish a baseline, the model also requires data to determine the level of maturity of the transportation system and its individual components. This is necessary in order to 
calculate the impact of levers at a later stage. Levers are new technologies or measures that are introduced to the system. Available levers either shift transport volume from a high to a low-emitting mode of transport or increase the efficiency of an individual or several modes.

The transportation system model supports the common material flows and indicators, covered by the ecoinvent database, but its focus is on greenhouse gases and regulated air pollutants. For levers only, the social dimension is addressed with the KPI local job creation potential of project related infrastructure build-up. As economic figures, CAPEX and OPEX of newly built infrastructure are calculated. The social and economic KPIs are not discussed in the following.

\subsection{Structure of the Model}

The model breaks down the general transportation demand within the city boundaries, with the functional units being passenger kilometres and ton kilometres. The main mode categories are rail based, road based and non-motorized transportation. The two functional units are broken down into vehicle types, where they are converted into actual vehicle kilometres, regardless of whether the mode is mass rapid transit by metro or individual passenger transport by car. The individual vehicle types are then specified in a parameterized process.

The same structure applies to the power model, which is connected to all processes, consuming electricity in the urban transportation system. The power is drawn on different grid levels, reflecting different T\&D losses and the grid mix is specified by fuel type and region.

Along with the transportation demand-oriented processes, a second stream quantifies basic road infrastructure such as lighting. The lighting demand is assessed based on lamp types and energy consumption and converted into lumen*h as a functional unit.

In order to model the set of 38 transportation and some energy levers, additional processes are modelled or the changes to the infrastructure are directly implemented in baseline processes. The calculations are each triggered by only one parameter and modelled on global (project) level or process level, depending on rather the induced change effects several processes or just one process.

\subsection{Parameterization}

The baselining process starts out with the model of a default city and adjusts it step by step to the actual city. The process can be compared to the fitting process of a tailor, making a dress on demand. Just like the dress, which starts with a certain size, but somewhat standard proportions, the city's transportation system model is assembled from different parts that are pre-configured with default values and 
assembled. In two to X fittings, data is adjusted to perfectly fit the city's transportation system or match its official reporting. The necessary fitting parameters are not hard coded, but part of questionnaire and fed to the model via calculation setups. The level of parameterization is the result of constant adaptations to the needs of numerous cities. In order to avoid having to feed thousands of parameters to the model for each calculation, most likely cases are defined, which can be combined and controlled with much fewer parameters. The hard-coded cases in the model consist of extracts from external databases such as HBEFA, which were imported via EcoSpold format previous to actual projects. This way, the number of commonly necessary data points was reduced to just 250 (not including forecasting parameters). Global project parameters define the scope of the model, which observation year to balance or the "flux" of transportation volumes of individual processes. Local process parameters are set to adapt individual processes. As one of the most relevant process categories, the mode type passenger cars for example are categorized by fuel type and conversion technology (combustion, hybrid...). Parameterization includes the average fuel consumption or tank to wheel GHG emissions, the EURO-Class, the difference in yearly vehicle mileage between diesel and petrol vehicles, as well as newer versus older vehicles and the average journey distance for cold start emission determination.

In addition to the parameters necessary for baselining, lever specific parameters are defined to obtain the current development or state of an individual infrastructure. Others identify the maximum applicability of a lever. One Parameter is set for each lever, to trigger it and set the degree to which it is implemented at a certain time.

\subsection{Forecasting}

The model is built as a decision support too for city administrations. When making long term infrastructure decisions, it is of little use to know impacts of a lever if it was in place today, if it takes 10 years in real world to construct the necessary infrastructure. The cause therefore requires forecasting options to determine the future baseline situation and lever impacts during the years the levers will actually be in place.

Forecasting elements are separated into three categories and treated differently:

- Forecasting parameters which are mainly influenced by the market development or can only be impacted by national or state union level governance are implemented by default or by case selection. This concerns technology improvements and fleet turnovers.

- Example 1: Average ages of vehicle technology are automatically forecasted based on vehicle fleet distributions in EURO classes. 
- Example 2: Future vehicle fleet distributions in EURO classes are automatically forecasted based on the current city's fleet distribution and matched with forecasts of the German national fleet.

- Parameter forecasts, which are the result of local phenomena such as the population increase or decrease and the travel behaviour, are extracted from reports or discussed in BAU-Scenario workshops with the city and have to be adjusted manually. MS Excel-based tools are provided to help with the effects of cross assumptions.

- Parameters impacted by local politics, facilitated in projects, policy and regulation are discussed in BAU-Scenario workshops and have to be modelled upfront in an iterative process, using levers, to determine input parameters.

- Example: If the city started constructing a new metro line, it is modelled in the transportation model and the resulting modal split in future years is used as inputs for the BAU-Scenario.

\subsection{Target Setting}

After the baseline is agreed in the form of a BAU scenario for the urban transportation system, the targets for different emissions at different target years are set. Carbon emission targets are commonly published, whereas air pollutant emission targets have to be retrieved from a separate process, converting limitations for concentrations of air pollutants at hot spot measurement stations into emission targets for transport-related emissions.

\subsection{Levers}

In order to reach their emission reduction targets, cities need to take measures. These measures are modelled as scenarios in the transportation system model and are referred to as levers. The levers aim at reducing transportation demand, shifting transportation volume to less polluting modes (modal shift), shifting to other fuels, increasing the energy/carbon efficiency of one or several modes of transport or adding pollution control devices. The levers can be of different natures (see the following list with examples):

- Technology based levers: advanced traffic light management

- Policy based: the implementation of a low emission zone

- A combination of policy and technology based: city tolling

- Behaviour change: eco-efficient driver training

- Generic: an X percent reduction of car use. 
The levers are implemented in the model as scenarios, with a ramp up phase, followed by another phase where they constantly deliver benefits. The level of possible implementation is defined by an implementation rate and depends on the current state and the maximum applicability. As an example: For the Lever Automated train operation (ATO) metro, the current state is the share of metro lines that have an ATO already and the maximum applicability would be $100 \%$ unless there are historic lines that shall keep human drivers.

The efficiency of each lever has to be quantified depending on the city specific conditions. And these conditions are assessed with individual parameters. In the case of the ATO for metros, a simulation in FALKO [3] (a simulation tool for train scheduling and simulation of energy consumption) was run for a typical metro line to check which parameters have the most significant impact on energy consumption. It turned out that the distance between two consecutive stations has the most significant correlation with the energy saving potential of an ATO. The city transport system model is therefore fed with the average distance between stations.

All levers model relative changes and can be combined in scenarios, also reflecting impacts of one lever on another lever. Some levers address the same saving potential and are therefore modelled combined in one process or on global/ project level.

\section{Results}

The following section states first the results in terms of how appropriate and practically feasible the above described methodology shows for the specific cause. Afterwards, the relevance of individual methodological aspects is illustrated in the form of actual modelling results from city projects.

\subsection{Scope}

The Scope of the model is in line with city's requirements. Two large cities requested two separate analyses for their inner city and outer city. Three cities, two of them in Scandinavian countries, requested the exclusion of Scope 3 GHG emissions in the energy upstream. Only one city in the south of Germany had Scope 3 GHG emissions from the energy upstream in their carbon accounting (WTW). All but two cities could be convinced to include Scope 3 GHG emissions for this relative comparison between levers. It allows a better symmetry between systems for indicators that are only of relevance in their global context. Since the studies did not aim to compare cities among each other, they did not have to fear an unfair competition.

It was a common request that for air pollutants, Scope 1 emissions should be reported individually. Especially traffic related urban emissions have a very high 
local impact due to the fact that they are emitted in very densely populated locations. Cities have strict local targets for these pollutants. Reporting on Scope 1-3 air pollution emissions is misleading in an urban context. In some cities for example in Austria, an increased share of photovoltaic (PV) power in the forecasted electricity mix lead to higher overall particulate matter emissions. These Scope 3 particulate matter emissions from PV panel production are of little relevance for the city where the panels are installed, since the emissions occur far away in much less densely populated areas and have little global impact.

\subsection{Structure and Parameterization}

The hierarchical structure of the model in combination with the strong parameterization involves difficulties in modelling practice, since a parameter calculated in a process is not passed down to processes in the lower hierarchies (upstream processes) in LCA-Software. Any parameter, used by more than one process, therefore has to be calculated on a project level (global parameter). LCA-software does not provide a folder structure on this level.

The most difficult parameters to derive are the overall transportation volume, especially for freight, and passenger kilometre or ton kilometre based modal split data. All cities were able to provide journey shares. But this data can't be used to create a passenger kilometre-based inventory without additional assumptions and data. Central databases such as Urban Audit asses KPIs, which are only a cutout of the overall transportation system. A typical example is the "[s]hare of journeys to work by car $-\%$ " [4].

\subsection{Forecasting \& Target Setting}

The first important information, the modelling delivers the cities, is how the overall sum of future emissions will develop over time in the BAU-Scenario. This serves as the baseline. Each lever calculation is compared with this BAU-Scenario to provide relative saving potentials. This is most relevant for $\mathrm{NO}_{2}$ (Fig. 1). It implies, that the distance to the city's target is significantly reduced before any lever is applied.

Secondly, the model delivers a contribution analysis in time slices. For PM2.5, emission shares of private vehicles increase compared to those of trucks (Fig. 2 vs. Fig. 3). 


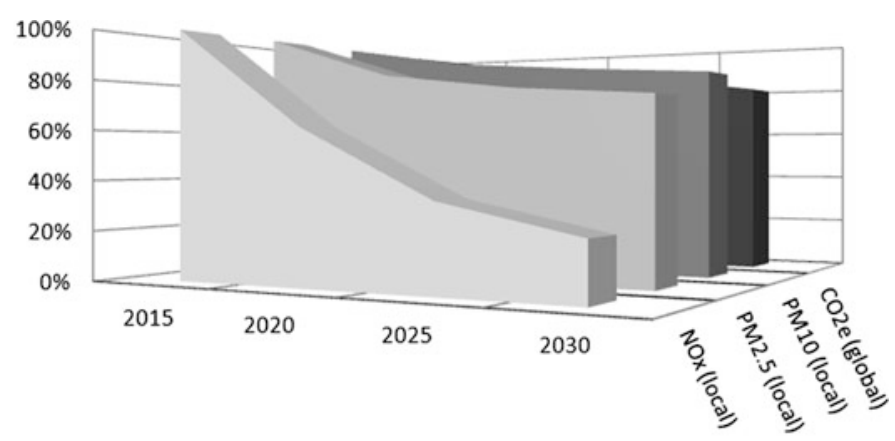

\begin{tabular}{|l|c|c|c|c|}
\cline { 2 - 5 } \multicolumn{1}{c|}{} & 2015 & 2020 & 2025 & 2030 \\
\hline m NOx (local) & $100 \%$ & $61 \%$ & $34 \%$ & $23 \%$ \\
\hline m PM2.5 (local) & $100 \%$ & $83 \%$ & $77 \%$ & $73 \%$ \\
\hline m PM10 (local) & $100 \%$ & $91 \%$ & $87 \%$ & $84 \%$ \\
\hline a CO2e (global) & $100 \%$ & $92 \%$ & $87 \%$ & $80 \%$ \\
\hline
\end{tabular}

Fig. 1 Relative reduction of four different transport related emissions in a city (results from a medium size city in the south of Germany)

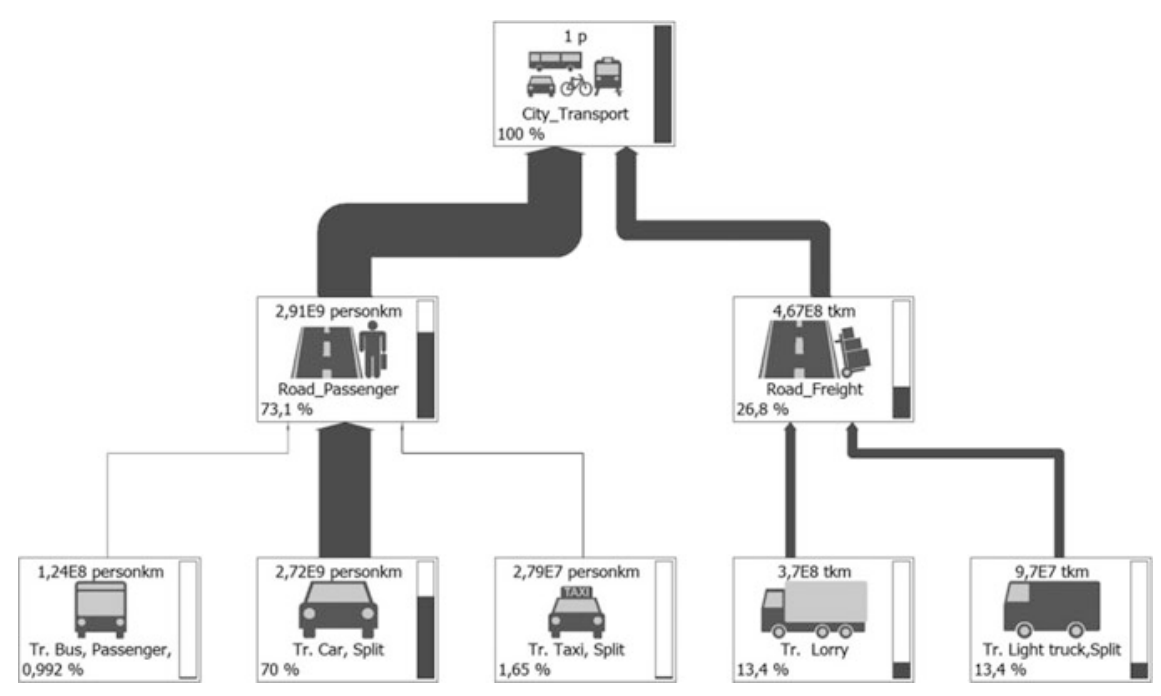

Fig. 22015 PM2.5 contribution tree (results from a medium size city in the south of Germany)

\subsection{Levers}

The third type of finding provides the lever analysis. For every lever, the new system performance of the city's transportation system at selected future years is calculated for GHG-Emissions (global) and for several air-pollutants (local). 


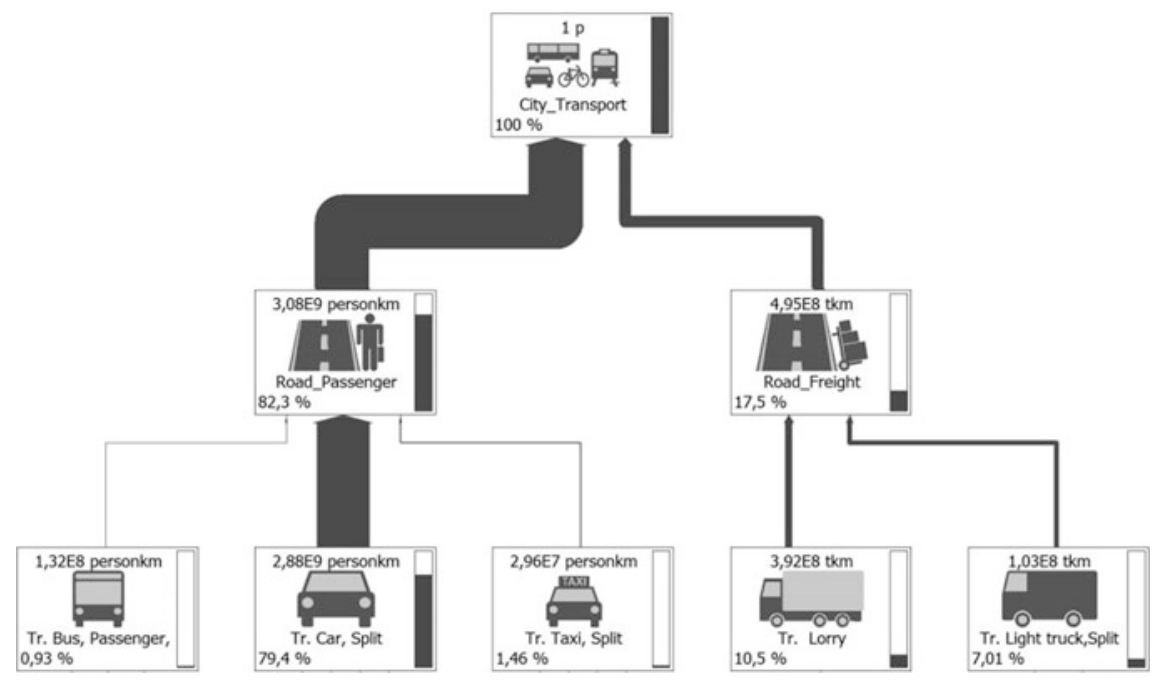

Fig. 32025 PM2.5 contribution tree (results from a medium size city in the south of Germany)

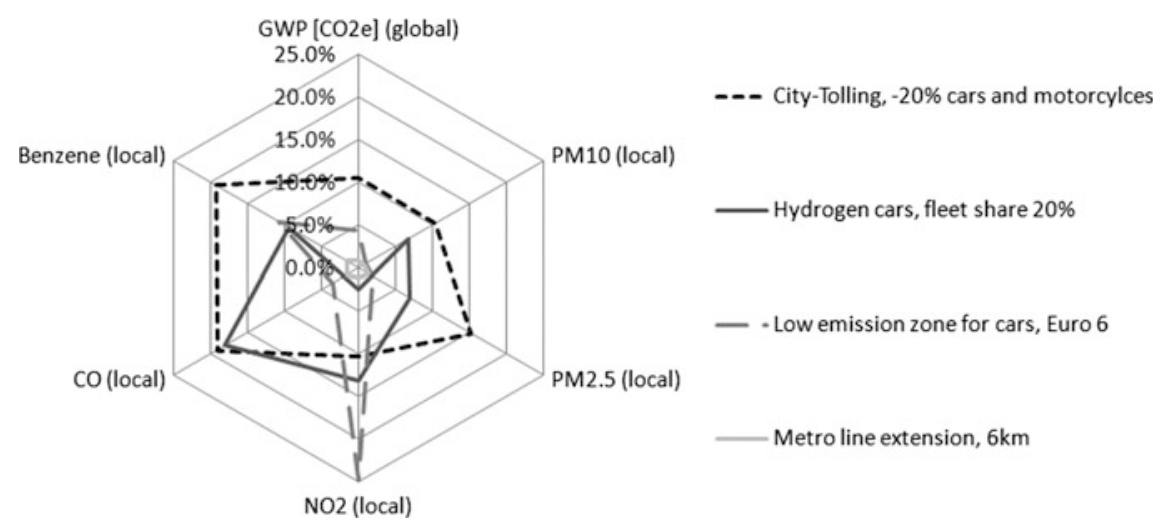

Fig. 4 Saving potentials of four levers for 6 different emissions at observation year 2025 (results from a medium size city in the south of Germany)

The following graph shows a spider diagram of a small selection of levers (Fig. 4). It clearly shows how diverse the impacts of a single measure can be on different indicators. 


\section{Interpretation}

\subsection{Scope, Structure and Parameterization}

The scope of the model must be flexible enough to adapt to any city's carbon reporting guidelines or methodology. It creates trust in the model, if results turn out similar to what the city expects to see for a carbon baseline. Based on this trust, additional scopes can be included for lever comparison. Since the target of this model isn't to compare cities, but levers within a city, the majority of all cities showed little reluctance to include additional scopes and therefore emissions.

The structural options of current LCA-Software provide limits to the complexity of models. Since parameters, calculated in one process can't be passed down to processes of subordinate processes, any cross-functionality between processes has to be modelled outside the processes on a project level, where it can't be properly managed. Since processes of urban transportation systems influence each other inherently, an increasing size of a model will eventually make it unmanageable in practice.

The overall transportation volume in pkm or tkm is not a KPI that is tracked by most cities. The determinations of the very basis of any urban transportation system balance, therefore becomes a tedious process.

\subsection{Forecasting and Target Setting}

The bottom up forecasting, based on commonly available emission databases, that have to be imported into the LCA-software, provide a good indication how emissions will develop in the future. For carbon, this is sufficient for target setting for lever selection and scenario definition, since carbon targets are expressed in annual emissions. For Air quality, it is a good indication, but hard to draw a final conclusion. A distance to political target/threshold judgment can only be made in combination with a contribution analysis of an individual air pollution measurement station, allowing an emission to concentration conversion. Some cities have this available already [5].

The contribution analysis of different time slices identifies which transportation mode to address with levers at what time, to get the maximum benefit. As an example: if a lever reduces the PM2.5 emissions of light trucks by 50\%, the effect for the overall transportation system related emissions will be almost twice as high in 2015 than in 2025 (see Figs. 2 and 3). 


\subsection{Levers}

The variety of measures that can be taken to improve an urban transportation infrastructure is very broad. Therefore, the impacts differ significantly on the chosen set of indicators, making a wider set of KPIs recommendable as a decision basis.

\section{Conclusion}

LCA-Methodology and tools are suitable to assess the transportation systems of a city and provide strategic decisions relevant information about alternative future developments.

A model, which is flexibility in scope and can adapt to any city's carbon accounting, is crucial in order to match existing reporting at least during the validation phase. This is true even if the final study later includes additional scopes. It is much easier and faster to check where differences are between the assessments. In addition, it allows the analysis of local effects such as air pollution.

Efficient model structuring and parameterization capabilities of LCA-software could be significantly improved if processes were capable of passing on parameters to subsequent processes.

Forecasting is necessary, when assessing systems that quickly change properties which effect target indicators and if the target is to compare systems that last long or take long to build. Both are the case for urban transportation systems, especially when focusing on air pollution. Otherwise absolute system emissions, as well as individual process contributions, have a high inaccuracy and might lead to wrong decisions.

The diversity in performance of a lever on different KPIs (Fig. 4) clearly shows that making an infrastructure decision for sustainable urban transport requires multi-KPI-modelling. This is a strength of LCA-methodology and software.

To significantly improve the applicability of LCA for urban transportation systems, KPI systems that cities report on should be adapted to assess indicators which cover the system as a whole and not only segments and can be used to create inventories.

\section{References}

1. Fong W, Sotos M, Doust M, et al., The Global Protocol for Community-Scale Greenhouse Gas Emission Inventories (GPC), 1st Ed., Greensouse Gas Protocol, 2014. < http://ghgprotocol. org/greenhouse-gas-protocol-accounting-reporting-standard-cities>, (Accessed 18.04.2017).

2. Keller M, Wuethrich P, Ickert L, Schmied M, Stutzer B, et al., Handbook Emission Factors for Road Transport, 3.2 Ed., INFRAS AG, 25.7.2014. 
3. Falko: Fahrplan-Konstruktion und -Validierung Optimierung betriebliche Planung und Steuerung, Siemens AG, 2007

4. Urban Audit, Eurostat, European Commission, 2016, < 2017http://appsso.eurostat.ec.europa. eu/nui/show.do?dataset=urb_ltran\&lang=en>, (Accessed 17.05.2017).

5. Rusp A, Verursacheranalyse 2013 für NOX, NO2 und PM10 für den Nürnberger LÜB-Standort Von-der-Tann-Straße, TÜV, 2014

Open Access This chapter is licensed under the terms of the Creative Commons Attribution 4.0 International License (http://creativecommons.org/licenses/by/4.0/), which permits use, sharing, adaptation, distribution and reproduction in any medium or format, as long as you give appropriate credit to the original author(s) and the source, provide a link to the Creative Commons license and indicate if changes were made.

The images or other third party material in this chapter are included in the chapter's Creative Commons license, unless indicated otherwise in a credit line to the material. If material is not included in the chapter's Creative Commons license and your intended use is not permitted by statutory regulation or exceeds the permitted use, you will need to obtain permission directly from the copyright holder.

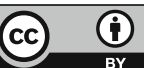

